\title{
Immunization-campaign reaction of Saudi Arabia citizens and residents during first six months of Coronavirus vaccine existence
}

\begin{abstract}
The COVID 19 vaccine provide acquired personal immunity against Coronavirus, its effectiveness is the risk of vaccinated participants compared with unvaccinated, reduces Coronavirus infections, efficacy preventing Coronavirus infections $95 \%$. The aim was to prove Coronavirus vaccine effectiveness during the first period six months, that was approved from WHO for KSA, as well immunization-campaign was started at 17/12/2020. So to clarify the extent of its impact on protecting the societal immunity of KSA society. The "Study Proficiency" as used on all persons in KSA, the "Intentional Physical" was prepared the questionnaires. All participants were $100 \%$ citizens and residents of KSA was showed the importance of the research topic in the KSA societal immunity. About $96 \%$ was agreed that the Coronavirus vaccine exists in KSA, 94\% was explained the extent feel protected from Coronavirus infections as a result of taking the Coronavirus vaccine. About half, $46 \%$ had received the Coronavirus vaccine, $94 \%$ was explained the importance of the Coronavirus vaccine for societal immunity protection and reducing Coronavirus infections in the KSA society. While $92 \%$ was urged those who refuse the Coronavirus vaccine to take it, $85 \%$ was found an improvement in the KSA societal immunity status of society. About $92 \%$ was helped to follow the societal immunity regulations of the Coronavirus vaccine. Showed $96 \%$ was confirmed the continuation of societal immunity precautions during the Coronavirus vaccine in the immunization-campaign for vaccination period. That of $84 \%$ was affirmed Coronavirus system should be pursued in other countries in order to reduce Coronavirus infections. That found $52 \%$ was advising to follow "Precautionary Health Strategy", 26\% was recommended the Coronavirus vaccine must taken to protect the individual and the KSA society, and $14 \%$ was protection by medication or therapeutic supplements such as vitamins. It was concluded that the Coronavirus vaccine had a clear effect on protecting the individuals health and societal immunity in the KSA society during first six months of Coronavirus vaccine existence. Despite the presence of a very small number of individuals rejecting the Coronavirus vaccine. It was recommend that the Coronavirus vaccine in the KSA had demonstrated its effectiveness, and therefore citizens and residents must take the Coronavirus vaccine to preserve the KSA individuals society health and societal immunity.
\end{abstract}

Keywords: COVID-19 vaccine, coronavirus, citizens and residents, KSA, ministry of health, precautionary health strategy

\author{
Volume 8 Issue 4 - 202I \\ Sherifa Mostafa M Sabra,' Afaf Bushara M \\ |smail $\left.\right|^{2}$ \\ 'Technology and Science Department, Ranyah University, KSA \\ ${ }^{2}$ Food and Nutrition Department, KSA
}

Correspondence: Sherifa Mostafa M. Sabra, Technology and Science Department, Ranyah University, Taif U, Saudi Arabia, EmailSh.sabra@tu.edu.sa/atheerl800@yahoo.com

Received: July 29, 202I | Published: August 23, 2021

\section{Introduction}

The COVID 19 vaccine provide acquired immunity against Coronavirus infections, that change expedited various vaccine application improvement during primal 2020. ${ }^{1}$ In Phase III trials, the vaccines incontestable efficacy $95 \%$ preventing Coronavirus infections. At 13 April 2021, the vaccines were authorized for national use, two RNA vaccines (Pfizer-BioNTech and Moderna), five recognized inactivated vaccines (BBIBP-CorV, CoronaVac, Covaxin, WIBP-CorV and CoviVac), four viral vector vaccines (Sputnik V, Oxford-AstraZeneca, Convidecia, and Johnson \& Johnson), and two protein subunit vaccines (EpiVacCorona and RBD-Dimer). ${ }^{2}$ The vaccine effectiveness is the risk of acquiring the Coronavirus infections by vaccinated individual compared with the unvaccinated individual risk. ${ }^{3}$ It reduces declaration and spread Coronavirus infections for fully vaccinated individual. ${ }^{4}$ Also it already gaining authorizations in globe countries to administered vaccinate individual required for societal immunity. ${ }^{5}$ That it had proposed as a possibly effective to cutoff Coronavirus infections pandemic, ${ }^{6}$ improvement industry to guarantee just access to these global countries immunizations. ${ }^{7}$ As well to activate and fund these global attempt against Coronavirus infections pandemic. ${ }^{8}$ The vaccine efficacy is $67 \%$ may be sufficiency to Coronavirus infections pandemic slow, award sterilizing societal immunity, necessary to Coronavirus infections prevention. ${ }^{9}$ That supply global countries about $60-70 \%$ global societal immunity. ${ }^{10}$ Public health and societal immunity agencies suggested vaccinated individual has more supported by preventative measures (face masks, social space and hands hygiene), particularly for open individual in the areas with high societal immunity spread. Governments indicated portray will be reduced as vaccination rates increase and societal immunity spread declines. ${ }^{11}$ The Coronavirus vaccine had granary immense global interest, less than a year after Coronavirus infections was alleged a pandemic. That are emerging in the fight are exceeding improvement, societal immunity odd at a national level. 
Also much more so for achieving at the world level, and straitening solutions implemented at standard. That will the global national societal immunity and health community end Coronavirus infections pandemic. ${ }^{8}$ The main aim of this research was to prove the effectiveness of the Coronavirus vaccine that was approved from WHO for KSA, as well immunization-campaign was started at 17/12/2020 during the first period, which was six months, in order to clarify the extent of its impact on protecting the KSA societal immunity and society health.

\section{Methodology}

The "Study Proficiency" was used on all individual in KSA, the "Intentional Physical" was prepared the questionnaires from Coronavirus vaccine started for the paper aims; (Table 1). ${ }^{12}$ The "Linked Appraisals" was used for the contented and study modesty. The "Reaching survey enquiry consequences" were assembled, then the "Modest Excel Bundle" was defined the consequence. ${ }^{13}$

Table I Questionnaire for immunization-campaign reaction of Saudi Arabia citizens and residents during first six months of Coronavirus vaccine existence

\begin{tabular}{|c|c|c|c|}
\hline \multirow[t]{2}{*}{ No. } & \multirow[t]{2}{*}{ Question } & \multicolumn{2}{|c|}{ Answer } \\
\hline & & Yes & No \\
\hline I. & \multicolumn{3}{|c|}{ Citizen or resident in KSA? } \\
\hline 2. & \multicolumn{3}{|c|}{ Agree for Coronavirus vaccine existence in KSA? } \\
\hline 3. & \multicolumn{3}{|c|}{ Feeling protected from Coronavirus infections after the vaccine availability KSA? } \\
\hline 4. & \multicolumn{3}{|c|}{ Received Coronavirus vaccine in KSA? } \\
\hline 5. & \multicolumn{3}{|c|}{ Confirm the Coronavirus vaccine should be taken for all individuals in KSA? } \\
\hline 6. & \multicolumn{3}{|c|}{ Urged to take Coronavirus vaccine for individuals refusing in KSA? } \\
\hline 7. & \multicolumn{3}{|c|}{ There is an improvement societal immunity situation after the presence of Coronavirus vaccine in KSA? } \\
\hline 8. & \multicolumn{3}{|c|}{ Supports the adoption of the Coronavirus vaccine system in KSA? } \\
\hline 9. & \multicolumn{3}{|c|}{ Confirms the continuation of "Precautionary Health Strategy" with Coronavirus vaccine period in KSA? } \\
\hline 10. & \multicolumn{3}{|c|}{ Want to follow the Coronavirus vaccine plan in other countries? } \\
\hline II. & \multicolumn{3}{|c|}{ Can you help us with advice during the period of Coronavirus vaccine in KSA } \\
\hline
\end{tabular}

\section{Results and discussion}

Table 2 \& graph 1 presented questionnaire results for immunization-campaign reaction of Saudi Arabia citizens and residents during first six months of Coronavirus vaccine existence, it was submitted via websites and the result was received in terms of the number of participants suitable for the research. It was found from the first question was "Citizen or resident in KSA?"; showed all participants were $100 \%$ citizens and residents of KSA, and this showed the importance of the research topic in the KSA societal immunity. ${ }^{1-2}$ The second question was "Agree for Coronavirus vaccine existence in KSA?"; that was indicated nearly $96 \%$ of participants agreed that the Coronavirus vaccine exists in KSA, and it was started given to the KSA societal immunity that was approved from WHO for KSA, as well immunization-campaign was started at 17/12/2020 including citizens and residents. ${ }^{1-2}$ The third question was "Feeling protected from Coronavirus infections after the vaccine availability KSA?"; $94 \%$ of the participants was explained the extent to which they feel protected from Coronavirus infections with the Coronavirus infections as a result of taking the Coronavirus vaccine, which protected individuals with a societal immunity and healthy protection from the Coronavirus infections. ${ }^{3-4}$ As for the fourth question was "Received Coronavirus vaccine in KSA?"; it was cleared that $46 \%$, about half of answered persons, had received the Coronavirus vaccine. That because the "Ministry of Health" systematized according to the age groups to administer the Coronavirus vaccine, and the number had reached almost half of residents and citizens. ${ }^{5-8}$ The fifth question was "Confirm the Coronavirus vaccine should be taken for all individuals in KSA?"; that was clarified the extent of the emphasis on taking the Coronavirus vaccine and it was about $94 \%$ of all. That which explained the importance of the Coronavirus vaccine for societal immunity and health protection and reducing Coronavirus infections in the KSA society. ${ }^{5-8}$ The sixth question was "Urged to take Coronavirus vaccine for individuals refusing in KSA?"; was clarified that $92 \%$ of the participants urged those who refuse the Coronavirus vaccine to take it to protect the individuals health and societal immunity, reduce Coronavirus infections, and maintain the KSA societal immunity and society health. ${ }^{3-4}$ The seventh question was "There is an improvement societal immunity situation after the presence of Coronavirus vaccine in KSA?"; was clarified that $85 \%$, or more than three quarters, found an improvement in the KSA societal immunity and health status of society during the first six months of the Coronavirus vaccine in the KSA society. This was evidence of the effectiveness of the Coronavirus vaccine in the performance societal immunity and societal immunity for individuals health protection and KSA society. ${ }^{9,10}$ The eighth question was "Supports the adoption of the Coronavirus vaccine system in KSA?"; that was found about $92 \%$ of all helped to follow the societal immunity and health regulations of the Coronavirus vaccine through advertisements, societal immunity and health programs, and also by dealing with members of health centers and the health sector in KSA, and this encouraged individuals to take the Coronavirus vaccine in an easy and accessible way. ${ }^{5-8}$ The ninth question was "Confirms the continuation of "Precautionary Health Strategy" with Coronavirus vaccine period in KSA?"; was identified $96 \%$ who were close to everyone. They confirmed the continuation of societal immunity and health precautions during the Coronavirus vaccine in vaccination period, as the Coronavirus vaccine works to produce antibodies within 15 days of taking the Coronavirus vaccine. 
So it had despite this they emphasized the measures strongly even for the individuals who took the Coronavirus vaccine because there were no new Coronavirus infections cases and reduce the occurrence of Coronavirus infections during gatherings or in the markets or family parties and others. ${ }^{8,11}$ It was found from the tenth question was "Want to follow the Coronavirus vaccine plan in other countries?"; that more than three-quarters of $84 \%$ was affirmed that the Coronavirus system should be pursued in other countries in order to reduce Coronavirus infections. Also restore businesses and others to their normal course to compensate for the losses incurred in KSA society and other countries during the period of high Coronavirus infections and the closure of commercial routes and others. ${ }^{5-8}$ That found through the participants the reaction was clear during the first six months of the Coronavirus vaccine in the KSA society, and the greater purpose was medical protection for individuals, reducing Coronavirus infections, protecting societal immunity for KSA society, returning life to normal, compensation and reducing material losses..$^{9-10}$

Table 2 \& graph I Questionnaire results for immunization-campaign reaction of Saudi Arabia citizens and residents during first six months of Coronavirus vaccine existence

\begin{tabular}{|c|c|c|c|c|c|c|c|c|c|c|}
\hline Item & Quest & & & & & & & & & \\
\hline & QI & Q2 & Q3 & Q4 & Q5 & Q6 & Q7 & Q8 & Q9 & Q10 \\
\hline Yes & $100 \%$ & $96 \%$ & $94 \%$ & $46 \%$ & $94 \%$ & $92 \%$ & $85 \%$ & $92 \%$ & $96 \%$ & $84 \%$ \\
\hline No & $0 \%$ & $4 \%$ & $6 \%$ & $54 \%$ & $6 \%$ & $8 \%$ & $15 \%$ & $8 \%$ & $4 \%$ & $16 \%$ \\
\hline
\end{tabular}

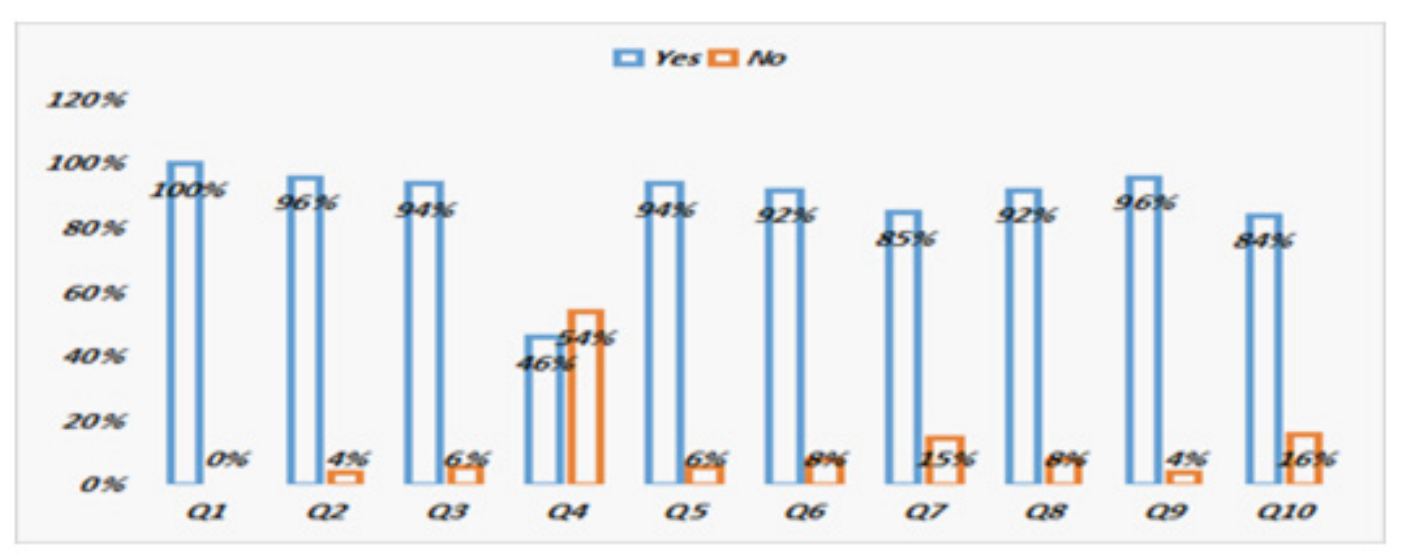

Table 3 \& graph 2 presented advice results for immunizationcampaign reaction of Saudi Arabia citizens and residents during first six months of Coronavirus vaccine existence, that was approved from WHO for KSA, as well as immunization-campaign was started at $17 / 12 / 2020$, it was found through the eleventh question was "Can you help us with advice during the period of Coronavirus vaccine in KSA"; for advice from the participating individuals, the highest advice was by more than half of the participants, $52 \%$ advising to follow "Precautionary Health Strategy". That such as washing hands well, using disinfectants, wearing a mask, not gathering and personal distancing, when there is a rise in temperature, you should go to the hospital or health center. Also not to hold celebrations or gatherings, not to crowd the markets, and to make personal and social distancing. ${ }^{8,11}$ The average advice was from more than a quarter of $26 \%$ who was recommended the Coronavirus vaccine must taken to protect the individual and the KSA society. ${ }^{3,4}$ The lowest advice was $14 \%$, which was protection by medication or therapeutic supplements such as vitamins. ${ }^{3,4}$ All the advice were good, but the less advice as well you should consult a Specialized Doctor in treatment and supplementation for the individual, as each individual differs from the other with societal immunity status. ${ }^{8,11}$ Through the questionnaire was conducted for the research, it was found that the Coronavirus vaccine was of societal immunity importance to the individual and society health. Through the interaction of the participants in the questions and advice, and the precautionary societal immunity conditions must be followed for all individuals to help reduce Coronavirus infections, enhance the societal KSA society societal immunity and strengthen of individuals status societal immunity. Thus, that are able to return to our normal life and could reduce the various losses of individuals and KSA society. $3,4,8,11$

Table 3 \& graph 2 Advice results for immunization-campaign reaction of Saudi Arabia citizens and residents during first six months of Coronavirus vaccine existence

\begin{tabular}{llll}
\hline Item & Advice & & \\
\hline & "Precautionary Health Strategy" & Coronavirus vaccine must taken & Protection by medication \\
Percent & $52 \%$ & $26 \%$ & $14 \%$ \\
\hline
\end{tabular}




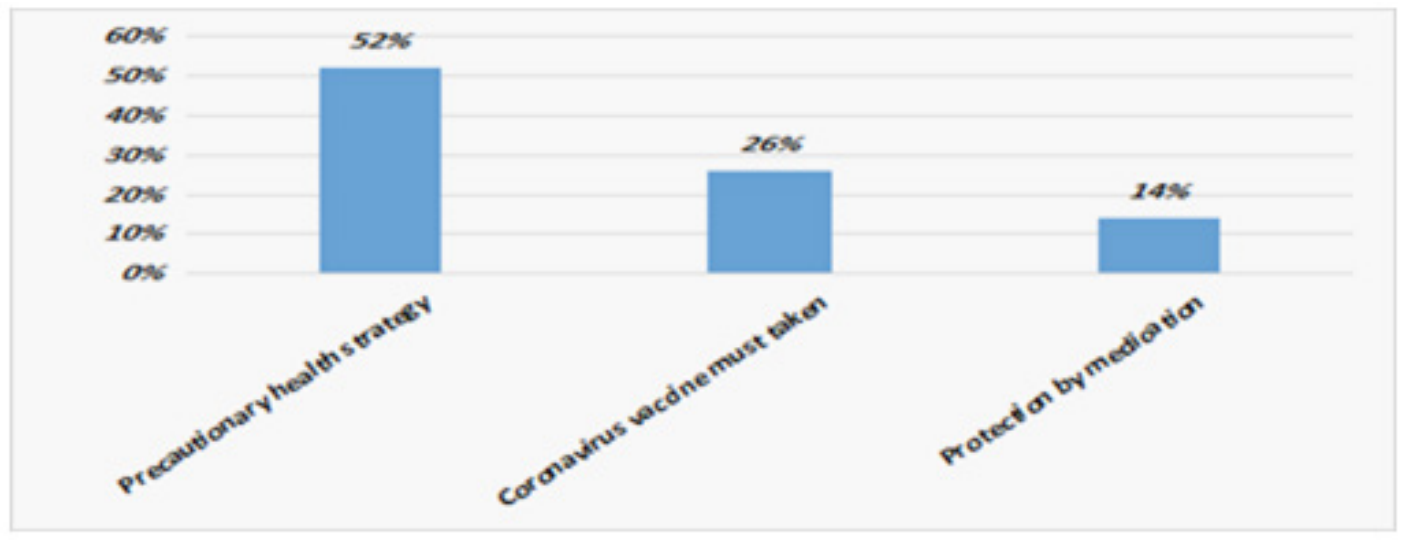

\section{Conclusion}

It was concluded that the Coronavirus vaccine had a clear effect on protecting the individuals health and societal immunity in the KSA society that from was approved from WHO for KSA, as well an immunization-campaign was started at 17/12/2020, despite the presence of a very small number of individuals rejecting the Coronavirus vaccine.

\section{Recommendation}

It was recommend that an immunization-campaign for Coronavirus vaccine in KSA had demonstrated its effectiveness, and therefore citizens and residents must take the Coronavirus vaccine to preserve the individuals health and KSA societal immunity for society health.

\section{Acknowledgments}

Sent thanks to the KSA citizens and residents whom the response was received for the effectiveness of Coronavirus vaccine and for assisting in the current research work.

\section{Conflicts of interest}

The author declares that there is no conflict of interest.

\section{Funding}

None.

\section{References}

1. Li Y, Chi Y, Su H, et al. Coronavirus vaccine development: from SARS and MERS to COVID-19. Journal of Biomedical Science. 2020;27(1):104.

2. COVID-19 vaccine development pipeline. Vaccine Centre, London School of Hygiene and Tropical Medicine. 2021.

3. Zimmer C. 2 Companies Say Their Vaccines Are 95\% Effective. What Does That Mean? You might assume that 95 out of every 100 people vaccinated will be protected from Covid-19. But that's not how the math works. The New York Times. 2020.
4. Centers for Disease Control and Prevention, (CDC). Interim Estimates of Vaccine Effectiveness of BNT162b2 and mRNA-1273 COVID-19 Vaccines in Preventing SARS-CoV-2 Infection Among Health Care Personnel, First Responders, and Other Essential and Frontline Workers, (PDF), Centers for Disease Control and Prevention (CDC). 2020

5. Wang Z, Schmidt F, Weisblum Y, et al. mRNA vaccine-elicited antibodies to SARS-CoV-2 and circulating variants. Nature. 2021. p. 1-7.

6. Forman R, Anderson M, Jit M, et al. Ensuring access and affordability through COVID-19 vaccine research and development investments: A proposal for the options market for vaccines. Vaccine. 2020;386075-6077.

7. World Health Organization, (WHO). COVAX Announces new agreement, plans for first deliveries. 2021.

8. Forman $\mathrm{R}$, Shah $\mathrm{S}$, Jeurissen $\mathrm{P}$, et al. COVID-19 Vaccine Challenges: What have we learned so far and what remains to be done?. Health Policy. 2021.

9. Randolph E, Barreiro B. Herd Immunity: Understanding COVID-19. Immunity. 2020;52(5):737-741.

10. McNeil D. Covid-19: how Much Herd Immunity is Enough? New York Times. 2021.

11. CDC. When You've Been Fully Vaccinated.. Centers for Disease Control and Prevention. 2021

12. Jirwe M, Gerrish K, Keeney S, et al. Identifying the core components of cultural competence: findings from a Delphi study. Journal of clinical nursing. 2009;18(18):2622-2634.

13. Purwanto A, Santoso B, Siswanto E, et al. Effect of Hard Skills, Sof Skills, Organizational Learning and Innovation Capability on Islamic University Lecturers' Performance. International Journal of Social and Management Studies. 2021;2(1):4-40. 